$\pm 0,10 \mathrm{~kg}$ ), chênh lệch $0,28 \mathrm{~kg}$, có YNTK $(p<0,05)$; sau 2 tháng $(0,93 \pm 0,3 \mathrm{~kg}$ so với 0,36 $\pm 0,17 \mathrm{~kg})$ chênh lệch $0,57 \mathrm{~kg}$, có YNTK $(p<0,01)$; chiều cao: chiều cao nhóm can thiệp có mức tăng tốt hơn nhóm chứng $(0,96 \pm 0,10 \mathrm{~cm}$ so với $0,59 \pm 0,17 \mathrm{~cm})$, chênh lệch $0,37 \mathrm{~cm}$, có YNTK $(p<0,05)$. Làm giảm tình trạng SDD và nguy cơ SDD: SDD thể nhẹ cân giảm $12,7 \%$ có YNTK $(p<0,05)$; nguy cơ SDD nhe cân giảm $14,5 \%$ có YNTK $(p<0,05)$. Không xuất hiện thừa cân, béo phì.

- Có xu hướng cải thiện tình trạng nhiễm khuẩn hô hấp, tiêu chảy, táo bón biếng ăn, khó ngủ: tỷ lệ mắc ở nhóm can thiệp thấp hơn so với nhóm chứng: nhiễm khuẩn hô hấp $(7,3 \% ; 9,1 \%)$; tiêu chảy $(6,4 \% ; 9,1 \%)$; táo bón $(4,5 \% ; 7,3 \%)$; biếng ăn $(10,9 \%$; $14,5 \%)$; khó ngủ $(7,3 \% ; 9,1 \%)$ nhưng số liệu còn ít chưa đủ làm kiểm định hoặc không có YNTK ( $p>0,05)$.

- Khả năng chấp nhận sử dụng cao: $96,3 \%$ số trẻ uống đủ khẩu phần sữa (2 bữa/ngày trong 2 tháng). Chương trình bổ sung sữa được gia đình, nhà trường, chính quyền, y tế ủng hộ.

\section{TÀI LIÊU THAM KHẢO}

1. Chính phủ (2015). Chiến lược quốc gia về dinh dưỡng giai đoạn 2011 - 2020 và tầm nhin đến năm 2030.

2. Chính phủ (2016). Chương trình sữa hoc đường cải thiện tình trạng dinh dưỡng góp phần nâng cao tầm vóc trẻ em mẫu giáo và tiểu học đến năm 2020.

3. Best C., Neufingerl N., Del Rosso J.M., et al. (2011). Can multi-micronutrient food fortification improve the micronutrient status, growth, health, and cognition of schoolchildren? a systematic review. Nutr Rev, 69(4), 186-204.

4. World Health Organization (2013). Essential Nutrition Actions: improving maternal, newborn, infant and young child health and nutrition.

5. World Health Organization (2007). The new WHO child growth standards. Bull World Heal Organ, 52(1), 13-17.

6. Flodin N.w. (1997). The metabolic roles pharmacology, and toxicology of lysine. J Am Coll Nutr, 16(1), 7-21.

7. Gombart A.F., Pierre $A_{\text {., }}$ and Maggini S. (2020). A Review of Micronutrients and the Immune System-Working in Harmony to Reduce the Risk of Infection. Nutrients, 12(1), 123-133.

\title{
SẢN XUẤT THUỐC BẰNG CÔNG NGHỆ IN 3D FDM PHẦN 2: CẢI THIỆN CHẤT LƯỢNG SẢN PHẨM IN 3D FDM
}

Lê Thị Thu Trang*, Trần Thị Hải Yến*, Võ Quốc Ánh*

\section{TÓM TẮT}

Trong những năm gân đây, công nghêe in 3D dựa trên nguyên lý FDM (fused deposition modeling) được nhiều nhà khoa học chú ý bởi những điểm sáng như khả năng ứng dụng rộng rãi và tiết kiệm chi phí. Tuy vậy, cũng giống như các kỹ thuật in 3D khác, chất lượng của sản phẩm FDM luôn là mối quan tâm lo ngai hàng đâu. Bài tổng quan này trình bày hai yếu tố chính, trực tiếp ảnh hưởng tới chất lượng của một vật thể in đó là nguyên vât liệu và thông số in. Bên canh đó, bài viết đề cập tới những nguyên nhân, mức độ ảnh hưởng của một số sư cố thường găp trong quá trình in 3D. Đồng thời, bài viết cũng tônng hợp những kinh nghiêm thành công khắc phuc sự cố kỹ thuât từ những nghiên cứu gân đây, tất cả cho một mục đích nâng cao chất lượng thuốc.

Từ khóa: Công nghệ in 3D, thuốc in 3D, cá nhân hóa điều trị, khắc phục sự cố trong in 3D.

\section{SUMMARY}

*Trường đại hoc Dước Hà Nôi

Chịu trách nhiệm chính: Võ Quốc Ánh

Email: anhvq@hup.edu.vn

Ngày nhận bài: 12.4.2021

Ngày phản biên khoa họ: 26.5.2021

Ngày duyệt bài: 14.6.2021

\section{QUALITY IMPROVEMENTS ON THE} PHARMACEUTICAL APPLICATION OF FUSED DEPOSITION MODELING 3D PRITING

Fused deposition modeling is a type of additive manufacturing technology that allows the production of three-dimensional (3D) devices from a computeraided design (CAD) file. FDM is an user-friendly, lowcost technique to quickly print prototypes with complex geometry. However, the FDM technique has some limitations due to its use of high temperatures. These temperatures may lead to potential thermal degradation, shrinkage issues, low surface quality and poor resolution. Moreover, although FDM is a fairly well-known 3DP technique, it is still a complex process to control. This complexity is due to the relatively high number of parameters that may affect the quality of final printed products. This paper aims to inform about 2 major factors that directly affect the quality of 3D printed products. Additionally, frequently encountered technical issues and approaches to solving the problems, improving product quality were also discussed.

Keywords: Three-dimension (3D) printing, 3D printed drugs, fused deposition modeling, material extrusion.

Từ ngữ viêt tăt: GPKD: Giải phóng kéo dài; $\mathrm{PCL}$ : polycaprolactone; HPMC: Hydroxypropyl Methylcellulose; HPC: hydroxypropyl cellulose; PEO: Polyethylene oxide; PEG: Polyethylene glycol; Kollidon ${ }^{\circledR}$ VA64: polyvinyl pyrrolidone vinyl acetate 64; 
HPMCAS :Hypromellose acetate succinate; PVP: Polyvinylpyrrolidone; EC: Ethyl cellulose; PVA: Polyvinyl acetate; PLA: polylactic acid; TEC: TriEthyl Citrate

\section{I. ĐẶT VẤN ĐỀ}

In 3D dựa trên nguyên lý FDM là công nghê bồi đắp từng lớp để tạo thành cấu trúc chi tiết 3 chiều của vật thể. Kỹ thuật này vượt trội hơn kỹ thuật bào chế truyền thống trong việc tạo ra các dạng bào chế phức tạp. Với tính linh hoạt, công nghệ này đã được ứng dụng để thiết kế và bào chế nhiều dạng thuốc khác nhau, từ các thuốc giải phóng nhanh đến các thuốc giải phóng có kiểm soát. Tuy nhiên, công nghệ này cũng có những hạn chế nhất định, một trong số đó là vấn đề chất lượng sản phẩm bao gồm chất lượng về mặt cảm quan, độ sắc nét của sản phẩm in; độ chính xác của sản phẩm so với thiết kế; đô ổn đinh về mặt hóa hoc, vật lý của dược chất; khả năng giải phóng của dược chất. Mặc dù FDM là một kỹ thuật in 3D khá nổi tiếng, nhưng đây vẫn là một kỹ thuật phức tạp, khó kiểm soát. Sư phức tạp này là do chất lượng của sản phẩm phụ thuộc vào rất nhiều yếu tố, từ những nguyên liệu cấu thành lên sợi in, sự thích hợp sợi in với máy in đến các thông số được cài đắt trong toàn bô quá trình sản xuất. Với muc tiểu cải thiện chất lượng thuốc in 3D FDM, bài viết này trình bày hai yếu tố chính, trực tiếp ảnh hưởng tới chất lượng của một vật thể in đó là nguyên vất liêu và thông số in. Bển canh đó, bài viết đề cập tới những nguyên nhân, mức độ ảnh hưởng của một số sự cố thường gặp trong quá trình in 3D. Đồng thời, bài viết cũng tổng hợp những kinh nghiệm thành công khắc phục sự cố kỹ thuật từ những nghiên cứu gần đây.
1. Môt số tá dược phổ biến được nghiên cứu ứng dụng trong FDM. Có thể thấy rằng, kỹ thuật in 3D FDM có nhiều hạn chế đối với các dược chất và tá dược kém bền với nhiệt do nguyên liệu tiếp xúc với nhiệt độ cao trong quá trình hoạt hoạt động. Tuy nhiên, yếu điểm này có thể được cải thiện bằng việc lựa chọn cẩn thận các thành phần trong công thức filament, thềm các chất hố trợ và cài đặt các thông số in phù hợp. Bên cạnh dược chất, sự lựa chọn tá dược đóng vai trò quan trọng, quyết định đển sự thành công của quá trình in $3 \mathrm{D}$ FDM, bởi vì cả dược chất và tá dược đều có ảnh hưởng trực tiếp tới tính lưu biến và các tính chất cơ học của sợi in. Một số polyme thích hợp sử dụng trong cổng nghệ FDM như PEO N10, HPMC E5, polycaprolactone và Mowiflex do có khả năng tao ra sợi in với độ cứng và tính chất lưu biến phù hợp, dễ dàng nạp vào máy in [1] [2]. Một số polyme như Eudragit ${ }^{\circledR} \quad E P O$, Soluplus ${ }^{\circledR}$ và Kollidon ${ }^{\circledR}$ VA64, HPMCAS EF, HPMCAS HF, EC cần thêm các tác dược khác, như chất làm trơn hay chất hóa dẻo, để làm giảm nhiệt độ nóng chảy và hạn chế hiện tượng gãy võ của filaments [1]. Ngoài sự ảnh hưởng tới khả năng in, polyme còn đóng vai trò quyết định đến khả năng giải phóng của dược chất. Thông thường, với polyme tan trong nước hoặc trương nở, dược chất sẽ giải phóng theo cơ chế khuếch tán và bào mòn. Với polyme không tan trong nước như EC, thuốc chủ yếu giải phóng theo cơ chế khuếch tán. Một số polyme và các chất phụ đã được ứng dụng trong bào chế thuốc bằng công nghệ in 3D FDM được trình bày trong Bảng 1 [3].

\begin{tabular}{|c|c|c|c|c|c|}
\hline $\begin{array}{l}\text { Dược chất (\% dược } \\
\text { chất trong } \\
\text { filament) }\end{array}$ & Polyme & $\begin{array}{c}\text { Tá dược } \\
\text { khác }\end{array}$ & $\begin{array}{c}\text { Nhiệt độ đun } \\
\text { filament tối } \\
\text { ưu }\end{array}$ & Nhiệt độ in & $\begin{array}{c}\text { Cơ chế giải } \\
\text { phóng } \\
\text { thuốc }\end{array}$ \\
\hline Paracetamol (30\%) & $\begin{array}{c}\text { HPC EC, HPMC, } \\
\text { Soluplus } \AA, \text { Eudragit } \AA \\
\text { L100 }\end{array}$ & $\begin{array}{c}\text { Kollidon } \\
\text { C-F }\end{array}$ & $140-180^{\circ} \mathrm{C}$ & $200^{\circ} \mathrm{C}$ & GPKD \\
\hline $\begin{array}{c}\text { Acetylsalicylic acid } \\
(5-15 \%)\end{array}$ & PCL & & $100^{\circ} \mathrm{C}$ & $100-120^{\circ} \mathrm{C}$ & GPKD \\
\hline Allopurinol (10\%) & PVA & Glycerol & $175^{\circ} \mathrm{C}$ & $200^{\circ} \mathrm{C}$ & GPKD \\
\hline Aripiprazol & PVA & & $172^{\circ} \mathrm{C}$ & $190^{\circ} \mathrm{C}$ & GP nhanh \\
\hline Baclofen (10\%) & PVA & Sorbitol & $160^{\circ} \mathrm{C}$ & $170-190^{\circ} \mathrm{C}$ & GPKD \\
\hline $\begin{array}{l}\text { Budesonid }(2.3 \%) \\
\text { Diclofenac }(20 \%) \\
\text { Theophyllin }(10 \%)\end{array}$ & PVP & $\begin{array}{c}\text { TEC; Talc; } \\
\mathrm{Na}_{3} \mathrm{PO}_{4}\end{array}$ & $160^{\circ} \mathrm{C}$ & $\begin{array}{l}185^{\circ} \mathrm{C} \text { khi in } \\
\text { thành viên } \\
110^{\circ} \mathrm{C} \text { khi in } \\
\text { lòng viên }\end{array}$ & GP ngắt nhịp \\
\hline Budesonid (5\%) & PVA & & $170^{\circ} \mathrm{C}$ & $190^{\circ} \mathrm{C}$ & GPKD \\
\hline Caffein (5\%) & Kollidon@ ${ }^{\circledR}$ VA64; PCL; & Kollidon $®$ & $140^{\circ} \mathrm{C}$ & $150^{\circ} \mathrm{C}$ & GPKD \\
\hline
\end{tabular}


VIETNAM MEDICAL JOURNAL N² - JUNE - 2021

\begin{tabular}{|c|c|c|c|c|c|}
\hline & PEO & P188 & & & \\
\hline $\begin{array}{c}\text { Caffein }(5 \%) \\
\text { Paracetamol(5-10\%) }\end{array}$ & PVA & & $180^{\circ} \mathrm{C}$ & $200^{\circ} \mathrm{C}$ & GPKD \\
\hline Calcein & PVA; PLA & & $210^{\circ} \mathrm{C}$ & $190^{\circ} \mathrm{C}$ & GPKD \\
\hline Carvedilol & HPMC & $\begin{array}{c}\text { Kolliphor@ } \\
\text { TPGS }\end{array}$ & $130^{\circ} \mathrm{C}$ & $135-200^{\circ} \mathrm{C}$ & GPKD \\
\hline $\begin{array}{c}\text { Ciprofloxacin (10- } \\
35 \%)\end{array}$ & PVA & $\begin{array}{c}\text { Dibutyl } \\
\text { sebacate }\end{array}$ & $175^{\circ} \mathrm{C}$ & $195^{\circ} \mathrm{C}$ & GPKD \\
\hline Domperidon (10\%) & HPC & $\begin{array}{l}\text { Barium } \\
\text { sulfate }\end{array}$ & $145-150^{\circ} \mathrm{C}$ & $210^{\circ} \mathrm{C}$ & GPKD \\
\hline Dronedaron $(10 \%)$ & PVA & PEG & $170^{\circ} \mathrm{C}$ & $200^{\circ} \mathrm{C}$ & GPKD \\
\hline Felodipin (10\%) & $\begin{array}{l}\text { Eudragit } ₫ \text { EPO } \\
\text { Soluplus } ₫ \text { PVA }\end{array}$ & \begin{tabular}{|} 
Tween 80; \\
PEG 4000; \\
PEO
\end{tabular} & $100-130^{\circ} \mathrm{C}$ & $150^{\circ} \mathrm{C}$ & $\begin{array}{l}\text { GPKD và GP } \\
\text { nhanh }\end{array}$ \\
\hline Furosemide (30\%) & 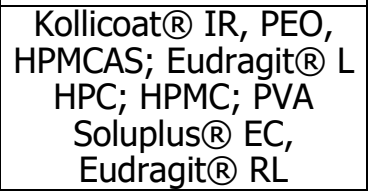 & $\begin{array}{c}\text { Glycerol; } \\
\text { PEG 400; } \\
\text { PEG 8000; } \\
\text { TEC }\end{array}$ & $65-190^{\circ} \mathrm{C}$ & $160-225^{\circ} \mathrm{C}$ & $\begin{array}{l}\text { GPKD; GP } \\
\text { nhanh và GP } \\
\text { ngắt nhịp }\end{array}$ \\
\hline $\begin{array}{l}\text { Haloperidol } \\
(10-20 \%)\end{array}$ & 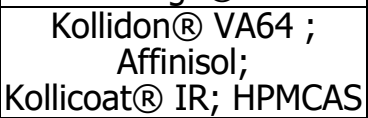 & & $170^{\circ} \mathrm{C}$ & $210^{\circ} \mathrm{C}$ & GP nhanh \\
\hline $\begin{array}{c}\text { Isoniazid } \\
(30 \%)\end{array}$ & $\begin{array}{c}\text { HPC; HPMC; PEO; } \\
\text { Eudragit } R \text { RL, RS and L }\end{array}$ & $\begin{array}{l}\text { Kolliphor@ } \\
\text { TPGS; TEC }\end{array}$ & $120-155^{\circ} \mathrm{C}$ & $165-195^{\circ} \mathrm{C}$ & $\begin{array}{c}\text { GPKD và GP } \\
\text { nhanh }\end{array}$ \\
\hline
\end{tabular}

2. Ảnh hưởng của thông số in 3D FDM tới chất lượng vật thể in

Kích thước fillament. Chất lượng sản phẩm in phụ thuộc nhiều vào sự đồng nhất trong filament và sự phù hợp giữa filament với máy in 3D. Ví dụ đối với máy in 3D MakerBot Replicator 2, filament có đường kính lý tưởng là 1,75 \pm 0,05 $\mathrm{mm}$. Đường kính quá nhỏ sẽ dễ bị bẻ gãy bởi bộ phận đùn và có thể tạo ra các bọt khí lẫn vào sản phẩm. Đường kính quá lớn sẽ gây lực cản lớn đến việc đẩy filament tới đầu in hoặc gây tắc đầu in. Để tạo ra được các sợi in tối ưu, cần sử dụng đầu in với đường kính phù hợp, dao động trong khoảng 1,3-3,0 mm. Để quá trình in liên tục và đồng đều, các filament cân có chiều dài dư sau in tối thiểu $20 \mathrm{~cm}$ và được uốn thành cuộn. Nhiều thiết bị hỗ trợ cho việc kéo sợi, làm mát, thu gom và cuộn filament đã được nghiên cứu và thử nghiệm (Hình 1) [4]

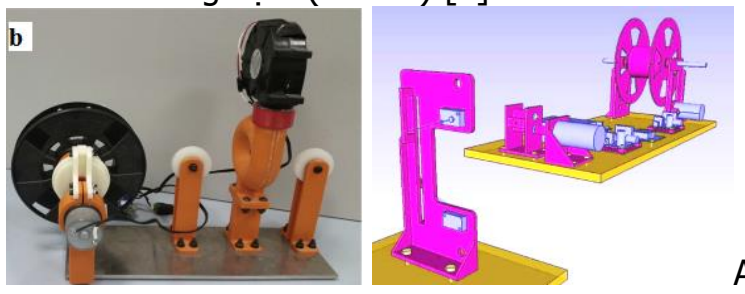

Hình 1: Một số thiêt kê hệ thống thu gom và cuôn filament cho máy in 3D FDM Kích thước đầu in (kim in)

Bên cạnh tính lưu biến và cơ học của sợi in, chất lượng sản phẩm được quyết định bởi các thông số cài đă̆t trong quá trình in 3D như tốc độ nạp sợi, nhiệt độ, đường kính đầu in và tốc độ di chuyển của đầu in. Trong đó, đường kính đầu in đóng vai trò quan trọng, ảnh hưởng tới nhiệt độ tối thiểu để quá trình in có thể diễn ra. Thồng thường, đường kính đầu in càng lớn, quá trình in có thể diễn ra ở nhiệt độ càng thấp. Ví dụ như khi in từ filament làm bằng $\mathrm{HPC} E F$ (hydroxypropyl cellulose EF), với đường kính đầu in $\phi=0,4 \mathrm{~mm}$ cần nhiệt độ in tối thiểu là $160^{\circ} \mathrm{C}$, trong khi đó với đường kính đâuu in $0,6 \mathrm{~mm}$ hoăc $0,8 \mathrm{~mm}$, chỉ cần $140^{\circ} \mathrm{C}$ [2]. Ngoài ra, kích thước đâu in còn ảnh hưởng tới sự thành công khi in các nguyên liệu có độ nhớt cao, thời gian in và độ sắc nét của sản phẩm.

Tốc độ di chuyển đâu in. Tốc độ di chuyển đầu in ảnh hưởng trực tiếp đến độ sắc nét của sản phẩm và thời gian in. Đầu in di chuyển với tốc độ quá nhanh có thể làm cho vật thể in bị méo do các lớp cũ chưa kịp đông rắn các lớp mới nóng chảy đã được thêm vào. Tuy vậy, khi in các vật thể ở dạng bản mỏng, tốc độ di chuyển đầu in ảnh hưởng không đáng kể đến chất lượng sản phẩm [5].

Nhiệt độ in. Là nhiệt độ được duy trì ở bộ phận gẩn đầu in, giúp làm nóng chảy filament. Nhiệt độ in ảnh hưởng tới độ nhớt, thể chất của mực in và do đó ảnh hưởng tới đặc tính của sản phẩm, quyết định sự thành công của quá trình in. Cần chọn nhiệt độ phù hợp, đủ để filament chảy lỏng, dễ dà̀ng được đẩy ra khỏi đầu in. 
Đồng thời, nhiệt độ không nên quá cao tránh làm phân hủy dược chất, vật liệu in không kịp đông rắn và biến dạng vật in. Ngoài ra, nhiệt độ in còn là yếu tố ảnh hưởing tới tính chính xác về kích thước, đô chính xác về cấu trúc vât thể in so với bản thiết kế. Đồng thời, đây là yếu tố ảnh hưởng tới khả năng giải phóng dược chất thông qua ảnh hưởng tới mức độ liên kêtt giữa các lớp in và liên kết giữa các phân tử polyme.

Độ lấp đây vật thể in. Đây là thông số quy định tỳ lệ không gian bên trong vật thể được lấp đầy bởi mực in, biểu thị độ đặc rỗng của sản phẩm. Đai lượng này có thể được cài đăt từ $0 \%$ (vật thể rỗng hoàn toàn) cho đến $100 \%$ (toàn bộ vật thể được in đặc khít). Độ lấp đầy ảnh hưởng tới thời gian in, tính chất cơ học của sản phẩm (độ cứng, độ dẻo). Với độ lấp đầy càng lớn, thời gian in càng dài và độ cứng sản phẩm càng cao
[6]. Độ lấp đầy thấp có thể giúp tiết kiệm nguyên liệu in và rút ngắn thời gian. Trong nghiên cứu thiết kế các dạng thuốc, độ lấp đầy có thể được dùng như là một yếu tố thay đổi cơ chế và khả năng giái phóng dược chất cũng như thay đổi tỉ trọng của dạng bào chế [7].

Góc nghiêng vật thể khi in. Thể hiện cách vật thể được in theo các trục $x, y$ hay $z$ (Hình $2 A)$. Yếu tố này ảnh hưởng tới thời gian in và độ bền cơ học của sản phẩm. Ví dụ như khi in sản phẩm hình hộp chữ nhật, thời gian in sẽ ngắn nhất khi in theo hướng $x$. Bên cạnh đó, góc nghiêng vật thể in ảnh hưởng tới độ cứng của sản phẩm. Trong một nghiên cứu năm 2013, Ashtankar và cộng sự đã chỉ ra rằng vật thể in từ ABS (Acrylonitril Butadien Styren) có độ cứng cao nhất khi in theo trục $x$ và giảm dần khi góc nghiêng tăng từ $0^{0}$ đến $90^{\circ}$.

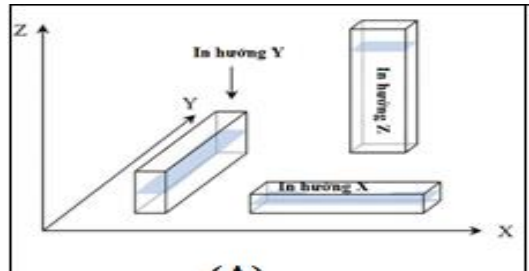

(A)
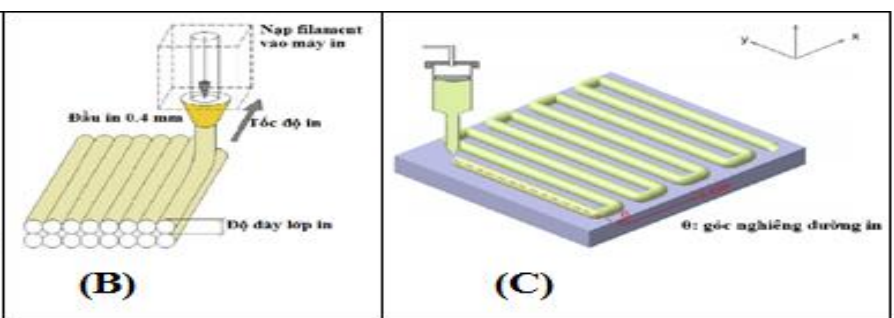

Hình 2: A: hướng in và góc nghiêng vật thể khi in (build orientation); B: độ dày lớp in (layer thickness); C: góc nghiêng đường in (raster orientation).

Độ dày lớp in. Là độ dày lớp in theo trục $z$ (Hình 2B), dao động trong khoảng 0,1-0,4 mm [8]. Thông thường, độ dày lớp in phải được cài đặt nhỏ hơn đường kính đầu in. Đây là yếu tố có ảnh hưởng tới độ cứng vật thể in. Thông thường, độ dày lớp in càng lớn, sản phẩm in có độ cứng càng cao. Cùng với nhiệt độ in, độ dày lớp in ảnh hưởng tới tính chính xác về kích thước vật thể in so với bản thiết kế. Do đó, để đảm bảo tính chính xác về kích thước, cấu trúc sản phẩm, nên cài đặt độ dày lớp in nhỏ. Có thể thay đổi độ dày lớp in bằng cách thay đổi thông số cài đặt hoặc thông qua chất liệu mực in, nhiệt độ và đường kính đầu in.

Độ dày lớp bao ngoài vật in. Là độ dày lớp ngoài cùng, bao xung quanh vật thể in, có thể có 1 lớp hoặc nhiều hơn. Độ dày lớp vỏ ảnh hưởng tới khối lượng riêng và độ cứng sản phẩm. Thông thường, lớp vỏ càng mỏng sẽ làm cho khối lượng riêng cũng như độ cứng vật thể càng nhỏ [7], [6]. Ngoài ra, độ dày lớp vỏ ảnh hưởng tới chất lượng bề mặt và độ chính xác về kích thước sản phẩm. Nghiển cứu của Perez năm 2018 cho thấy khi giảm độ dày lớp màng bao sẽ giảm được độ nhám, thô ráp trên bề mặt, sản phẩm có chất lượng cảm quan tốt hơn. Trong nghiên cứu bào chế thuốc, số lớp bao ngoài có ảnh hưởng lớn đến độ hòa tan dược chất thông qua kiểm soát khả năng khuếch tán của môi trường vào trong lòng viên.

Góc nghiêng đường in. Là góc tạo bởi đường in và trục $x$ trong hệ tọa độ ào xác định bởi máy in (Hình 2C). Nó ảnh hưởng đến góc tạo bởi đường in và bề mặt của vật in. Khi góc này bằng $90^{\circ}$, độ cứng của vật in đo được theo phương trục x là nhỏ nhất và độ cứng này sẽ lớn nhất khi góc nghiêng đường in bằng $0^{\circ}$.

\section{Những vấn đề kỹ thuật có thể gặp} phải trong in 3D và giải pháp. Tuy có cấu tạo và nguyên tắc hoạt động tương đối đơn giản, nhưng trong quá trình in nhiều sự cố có thể xuất hiện, ảnh hưởng tới quá trình in và chất lượng sản phẩm. Các sự cố này có thể xuất phát từ thiết bị, nhưng phần lớn bắt nguồn từ chất lượng của filament và sự phù hợp giữa các thông số của quá trình in. Những nguyên nhân, mức độ ảnh hưởng và cách khắc phục một số sự cố thường gặp trong quá trình in 3D FDM được trình bày trong Bảng 2 [3]. 
Bảng 2: Những vấn đề kỹ thuật có thể gặp phải trong in 3D FDM và các giải pháp

\begin{tabular}{|c|c|c|c|}
\hline $\begin{array}{l}\text { Vấn đề kỹ } \\
\text { thuât }\end{array}$ & Nguyên nhân & Mức độ ảnh hưởng & Cách khắc phục \\
\hline Tắc đâu in & $\begin{array}{l}\text { - Nguyên liệu bị phân hủy. } \\
\text { - Nguyên liẹu phân bố không } \\
\text { đều, có các tiếu phân lớn. } \\
\text { - Mực in có độ nhớt quá cao } \\
\text { - Filament bị gãy. }\end{array}$ & $\begin{array}{l}\text { Ảnh hưởng nghiêm } \\
\text { trọng, khiến quá trình } \\
\text { in bị gián đoạn. }\end{array}$ & $\begin{array}{l}\text { - Điêu chỉnh nhiệt độ in } \\
\text { - Thêm các tá dược trơn. }\end{array}$ \\
\hline $\begin{array}{l}\text { Bề mắt thô } \\
\text { ráp. }\end{array}$ & Độ nhớt mực in quá cao & $\begin{array}{l}\text { Mức độ ảnh hưởng } \\
\text { thấp, chủ yếu tới hình } \\
\text { thức sản phẩm. } \\
\end{array}$ & $\begin{array}{l}\text { - Giảm tốc độ di chuyến đầu in } \\
\text { - Tăng nhiệt độ in } \\
\text { - Thêm chất hóa dẻo }\end{array}$ \\
\hline $\begin{array}{l}\text { Độ phân giải } \\
\text { thấp, không } \\
\text { sắc nét }\end{array}$ & & $\begin{array}{l}\text { Ảnh hưởng tới hình } \\
\text { thức của sản phẩm }\end{array}$ & - Dùng đâu in nhỏ hơn \\
\hline $\begin{array}{l}\text { Tạo sợi tơ } \\
\text { xung quanh } \\
\text { chi tiết in. }\end{array}$ & Độ nhớt mực in quá thấp & $\begin{array}{l}\text { Ảnh hưởng tới hình } \\
\text { thức của sản phẩm }\end{array}$ & $\begin{array}{l}\text { - Giảm nhiệt độ đầu in } \\
\text { - Tối ưu đướng di chuyển đầu in } \\
\text { - Tăng tốc đô̂ và mức độ rút } \\
\text { filament. }\end{array}$ \\
\hline $\begin{array}{l}\text { Liên kết giữa } \\
\text { các lớp in } \\
\text { không tốt }\end{array}$ & $\begin{array}{l}\text { - Filament quá dẻo } \\
\text { - Nhiệt độ in thấp }\end{array}$ & $\begin{array}{c}\text { Mức ảnh hưởng } \\
\text { nghiêm trọng, ảnh } \\
\text { hưởng tới cơ chế giải } \\
\text { phóng thuốc }\end{array}$ & $\begin{array}{l}\text { - Tăng nhiêt độ đầu in, bàn in } \\
\text { - Giảm tốc độ quât làm mát } \\
\text { - Giảm tỷ lệ chất hóa dẻo } \\
\text { - Tăng tốc độ in } \\
\text { - Thêm mốt số chất tăng kết } \\
\text { dính như triacetine }\end{array}$ \\
\hline $\begin{array}{l}\text { Sản phẩm bị } \\
\text { cong, vênh. } \\
\text { Vật in không } \\
\text { vững }\end{array}$ & $\begin{array}{l}\text { - Liên kết vật - bàn in kém } \\
\text { Nguyên nhân có thể kể đến } \\
\text { là: } \\
\text { - Bề măt vất in quá nhỏ } \\
\text { - Lớp in đầu tiên bị co ngót } \\
\text { do làm mát quá nhanh }\end{array}$ & $\begin{array}{l}\text { Mức độ ảnh hưởng } \\
\text { nghiêm trọng }\end{array}$ & $\begin{array}{c}\text { - Tăng nhiệt độ bàn in, diện tích } \\
\text { tiếp xúc giữa vật in với bàn in } \\
\text { - Tăng độ dày lớp in đâu } \\
\text { - Giảm tốc độ di chuyển đâu in } \\
\text { - Giảm tốc độ quạt làm mát } \\
\text { vật in }\end{array}$ \\
\hline $\begin{array}{l}\text { Vật in bị méo, } \\
\text { sai lệch so với } \\
\text { mô hình }\end{array}$ & $\begin{array}{r}\text { - Đông rắn chưa ho } \\
\text { - Thời gian đông rắn } \\
\text { - Nhiệt độ in quá }\end{array}$ & $\begin{array}{c}\text { Ánh hưởng nghiêm } \\
\text { trọng }\end{array}$ & $\begin{array}{l}\text { - Tăng tốc độ quạt làm mát } \\
\text { - Giảm nhiệt đố phòng in } \\
\text { - Cải tiển filament }\end{array}$ \\
\hline $\begin{array}{l}\text { Filament bị } \\
\text { gãy khi in }\end{array}$ & Filament quá giòn. & $\begin{array}{r}\text { Anh hức } \\
\text { trọng, kh } \\
\text { in bị c }\end{array}$ & $\begin{array}{l}\text { - Thêm chất hóa dẻo vào } \\
\text { filament [2] }\end{array}$ \\
\hline $\begin{array}{l}\text { Filament bị } \\
\text { biến dạng }\end{array}$ & Filament quá dẻo & $\begin{array}{r}\text { - Anh hức } \\
\text { trọng, khié } \\
\text { in bi gi gi }\end{array}$ & - Giảm tỷ lệ chất hóa dẻo \\
\hline $\begin{array}{l}\text { Dược chất bị } \\
\text { phân hủy }\end{array}$ & Nhiệt độ in quá cao & $\begin{array}{l}\text { Mức độ ảnh hưởng } \\
\text { nghiêm trọng. }\end{array}$ & $\begin{array}{l}\text { - Tối ưu hóa nhiệt độ in. } \\
\text { - Tối ưu hóa công thức } \\
\text { filament }\end{array}$ \\
\hline $\begin{array}{l}\text { Filament bị } \\
\text { bào mòn, tốc } \\
\text { độ cấp bị sai }\end{array}$ & $\begin{array}{l}\text { Filament không đủ đô cứng, } \\
\text { dễ bị bào mòn bởi bánh răng } \\
\text { đẩy filament vào đâu in }\end{array}$ & $\begin{array}{l}\text { Ảnh hưởng nghiêm } \\
\text { trọng đến độ chính } \\
\text { xác, độ đồng đều. }\end{array}$ & $\begin{array}{l}\text { - Tăng nhiệt độ in } \\
\text { - Tăng tá dược làm cứng } \\
\text { filament } \\
\text { - Dùng đầu in lớn hơn }\end{array}$ \\
\hline $\begin{array}{l}\text { Tốc độ ra } \\
\text { mực không ổn } \\
\text { định }\end{array}$ & $\begin{array}{c}\text { Filament không đồng nhất, } \\
\text { bề mặt không nhắn, ma sát } \\
\text { lớn với ống dấn }\end{array}$ & $\begin{array}{l}\text { Ánh hưởng nghiêm } \\
\text { trông đến độ chính } \\
\text { xác, độ đồng đều. }\end{array}$ & Cải tiến chất lượng filament \\
\hline $\begin{array}{l}\text { Bọt khí sinh ra } \\
\text { trong quá } \\
\text { trình in }\end{array}$ & $\begin{array}{c}\text { Filament nhỏ, có săn bọt khí, } \\
\text { độ ẩm cao hoăc chứa các } \\
\text { dung môi tồn dư }\end{array}$ & $\begin{array}{l}\text { Mức độ ảnh hưởng } \\
\text { nghiêm trọng. }\end{array}$ & $\begin{array}{l}\text { - Chọn đầu in phù hợp. } \\
\text { - Loại khí, dung môi và ầm } \\
\text { trong filament }\end{array}$ \\
\hline
\end{tabular}

\section{KẾT LUÂNN}

Thuốc in 3D theo công nghê FDM có nhiều tiềm năng ứng dụng trong lĩnh vực Dược phẩm sản xuất các thuốc cá nhân hóa, các dạng thuốc có cấu trúc phức tạp, kiểm soát giải phóng. Tuy nhiên, để tiến xa hơn nữa, đưa công nghệ in 3D 
FDM vào thực tiễn thì viêc kiểm soát và nâng cao chất lượng sản phẩm là vô cùng quan trọng. Nguyên vật liệu, thành phần trong công thức thuốc cần được lựa chọn cẩn thận. Một phần để đảm bảo chất lượng sản phẩm đầu ra. Măt khác, không kém phần quan trọng, giúp đảm bảo quá trình in được diễn ra thuận lợi và thông suốt. Song song với đó, các thông số in như nhiệt độ, tốc độ in, độ dày lớp in, góc nghiêng đường in, góc nghiêng vật in, độ lấp đầy, bề dày lớp vỏ... cũng cần được tối ưu hóa nhằm tăng tính khả thi và cải thiện chất lượng sản phẩm.

\section{TÀI LIẸU THAM KHẢO}

1. Zhang, J., et al. (2019). "Development and evaluation of pharmaceutical 3D printability for hot melt extruded cellulose-based filaments.". Journal of drug delivery science and technology, 52, 292.

2. Henry, S., et al. (2021). "Extrusion-based $3 D$ printing of oral solid dosage forms: Material requirements and equipment dependencies.". International journal of pharmaceutics, 598, 120361.
3. Cailleaux Sylvain, e.a. (2020). "Fused Deposition Modeling (FDM), the new asset for the production of tailored medicines." Journal of Controlled Release

4. Melocchi, A., et al. (2015). "3D printing by fused deposition modeling (FDM) of a swellable/erodible capsular device for oral pulsatile release of drugs.". Journal of Drug Delivery Science and Technology, 30, 360-367.

5. Solomon, I.J., P. Sevvel, and J. Gunasekaran. (2021). "A review on the various processing parameters in FDM." Materials Today: Proceedings, 37, 509-514.

6. Zhang, J., et al. (2020). "Structure-function correlation and personalized 3D printed tablets using a quality by design (QbD) approach.". International Journal of Pharmaceutics 590, 119945.

7. Vo, A.Q., et al (2020). "Hot melt extrusion paired fused deposition modeling 3D printing to develop hydroxypropyl cellulose based floating tablets of cinnarizine." Carbohydrate Polymers, 246

8. Mitsouras, D., and Peter C. Liacouras. (2017). 3D printing technologies. In $3 \mathrm{D}$ printing in medicine, 5-22.

\section{ĐẶC ĐIỂM DI ỨNG VÀ KẾT QUẢ NHẬN DANG THUỐC CHỐNG LAO HÀNG 1 GÂY DI ỨNG TRÊN DA BẰNG TEST KÍCH THÍCH}

\section{TÓM TẮT}

Tổng quan: Cần phát hiện sớm các triệu chứng của dị ứng và có biện pháp xử lý kịp thời để tối ưu hóa vieêc điều trị thuốc chống lao. Mục tiêu: Mô tả đặc điểm di ứng và kết quả nhân dạng thuốc chống lao hàng 1 gây dị ứng trên da bằng test kích thích tại bệnh viện Phổi Trung Ương. Phương pháp: nghiển cứu mô tả cắt ngang 86 bệnh nhân lao phổi có chẩn đoán dị ứng thuốc lao, nhập viện tại khoa Lao hô hấp bệnh viện Phổi Trung ương từ tháng 8/2018 đển tháng 6/2019. Loại trừ bệnh nhân sốc phản vệ, dị ứng nặng, hội chứng SJS, Lyell, DRESS; có tình trạng co thắt đường thở không kiêm soát (FEV1<70\%). Kêt quả: 48 bệnh nhân $(55,8 \%)$ biểu hiện mày đay, ban chấm; 85 bệnh nhân $(98,8 \%)$ biểu hiện ngứa. Tổn thương da: $55 \%$ mức độ $2,39 \%$ mức độ 3 và $6 \%$ mức độ 4. Điêu trị trước khi test kích thích: số ngày điều trị trung bình 4,3 ngày (1-16 ngày), $33,7 \%$ số trường hợp phải sử dụng cả thuốc kháng histamin và corticoid. Có 81 bênh nhân đước test kích thích với tổng số 346 lần testi, trong đó có 80 lần test kích thích cho kết quả dương tính. Thuốc có tỉ lệ dị ứng từ cao

\footnotetext{
${ }^{1}$ Bệnh viện Phổi Trung ương

${ }^{2}$ Đại học Y Hà Nọi

Chịu trách nhiệm chính: Hoàng Tuấn

Email: hoantuan@hmu.edu.vn

Ngày nhận bài: 19.4.2021

Ngày phản biện khoa học: 28.5.2021

Ngày duyệt bài: 16.6.2021
}

, Nguyễn Kim Cương1,2, Nguyễn Mạnh Thế

đến thấp lần lượt RIF, PZA, EMB, INH. Có 54 bệnh nhân dị ứng với chỉ 1 loại thuốc, 13 bệnh nhân dị ứng với 2 loại thuốc, không có trường hợp nào di ứng với từ 3 thuốc trở lên. Đa số bệnh nhẩn test kích thích dương tính vào ngày thứ 2 hoặc thứ 3 . Kết luận: Phác đồ điều trị bệnh lao gồm nhiều thuốc phối hợp do đó khi xảy ra phản ứng di ứng rất khó khăn trong chẩn đoán thuốc gây ra phản ứng. Tuy nhiên khi thực hiên test kích thích, lần lượt từng thuốc được tiến hành do đó có thể xác định nguyên nhân gây dị ứng môt cách chính xác

Tư khóa: dị ứng thuốc lao, ADR, test kích thích

\section{SUMMARY}

\section{ALLERGIC CHARACTERISTICS AND RESULTS OF PROVOCATION TEST IN PATIENTS WITH ANTI-TUBERCULOSIS DRUG ALLERGY}

Background: Early detection of hypersensitivity reactions and appropriate management should be taken to optimize the treatment of tuberculosis. Objective: Description of hypersensitivity reactions from first-line antituberculosis drugs and results of drug provocation test at National Lung Hospital. Methods: Cross-sectional study of 86 pulmonary tuberculosis patients with signs and symtomps of hypersensitivity reactions, who were hospitalized and treated in the Department of Respiratory Tuberculosis at National Lung Hospital from August 2018 to June 2019. Excluded from the study were patients with anaphylaxis, severe allergy, SJS, Lyell syndrome, 